\title{
TeV J2032+4130 - very high energy gamma-ray source of unresolved nature
}

\author{
V.G. Sinitsyna ${ }^{1, *}$, V.Y. Sinitsyna ${ }^{1}$, K.A. Balygin ${ }^{1,2}$, S.S.Borisov ${ }^{1}$, A.M. Kirichenko ${ }^{1,2}$, A.I. Klimov ${ }^{1}$, \\ R.M. Mirzafatikhov ${ }^{1}$, N.I. Moseiko ${ }^{1,2}$, I.E. Ostashev ${ }^{1,2}$ \\ ${ }^{1}$ P. N. Lebedev Physical Institute, Russian Academy of Sciences \\ ${ }^{2}$ National Research Center "Kurchatov Institute"
}

\begin{abstract}
The Cygnus Region is one of the brightest regions in all ranges of the electromagnetic spectrum and contains a number of potential $\mathrm{GeV}$ and $\mathrm{TeV}$ emission sources. It includes active star formation regions, pulsars and supernova remnants. Some of the sources have been detected at high and very high energies. One of them discovered due to its proximity to the well-known microquasar Cyg X-3 is the object TeV J2032+4130. This object is still of unresolved nature and is being intensively studied in different energy ranges. The results of twenty-year observations of TeV J2032+4130 by the SHALON experiment are presented in this paper. The collected experimental data on fluxes, spectrum shape and morphology of TeV J2032+413 can help in the future to determine an object type and reveal mechanisms of generation of very high energy emission.
\end{abstract}

\section{Introduction}

The Cygnus Region is one of the brightest areas of the sky in all wavelengths and is known to contain a number of potential $\mathrm{GeV}$ and $\mathrm{TeV}$ gamma-ray sources. Objects connected to this region include star association Cygnus OB2, the microquasar Cygnus X-3, supernova remnant Cygni SNR and the TeV J2032+4130 object. Some of the sources listed above have been detected at high energies with the Fermi LAT experiment [1-3] and earlier with the EGRET telescope $[4,5]$. Also, it was detected at very high energies with the Milagro air shower array detector [6] and by imaging atmospheric Cherenkov telescopes Whipple [7], HEGRA [8], SHALON [9-11], VERITAS [12] and MAGIC [13].

TeV J2032+4130 was detected by the HEGRA telescopic system [8] at energies $>1 \mathrm{TeV}$ during observations made in the years 1999 - 2001. This object was the first $\mathrm{TeV}$ gamma-ray detection having no bright counterpart at any other wavelength and was also the first extended source to be discovered in the very high energy range. Characteristics of $\mathrm{TeV} \mathrm{J2032+4130} \mathrm{in} \mathrm{the} \mathrm{energy} \mathrm{range}$ from 1 to $50 \mathrm{TeV}$ were obtained with the SHALON telescope for the first time. The observation data on the TeV $\mathrm{J} 2032+4130$ object presented in this paper are the result of twenty-year-long investigations of the Cygnus Region by the SHALON experiment.

\section{TeV J2032+4130}

In accordance with the program on long-term studies of the microquasar Cygnus X-3 [9-11] at very high energies, observations of the Cygnus Region and its member objects, including $\mathrm{TeV} \mathrm{J} 2032+4130$ are being carried out

\footnotetext{
*e-mail: sinits@sci.lebedev.ru
}

with the high-mountain SHALON imaging atmospheric Cherenkov telescopes. TeV J2032+4130 is located at a distance of $\sim 0.5^{\circ}$ north from Cyg X-3. So due to the large telescopic field of view $>8^{\circ}$ the observations of Cygnus-X are naturally followed by tracking TeV J2032+4130.

As a result, TeV J2032+4130 as a source accompanying Cyg X-3 has been systematically observed with the SHALON telescope (since 1995 up to now) during clear moonless nights at zenith angles from $5^{\circ}$ to $34^{\circ}$. The observations were performed using the standard SHALON technique of recording information about the cosmic-ray background and gamma-ray-initiated showers in the same observing session [14-16]. With the data processing, TeV J2032+4130 was detected above $0.8 \mathrm{TeV}$ by SHALON with a statistical significance $31 \sigma$ determined by the Li\&Ma method [17]. The average integral flux at energies above $0.8 \mathrm{TeV}$ for $\mathrm{TeV} \mathrm{J} 2032+4130$ is $I_{T e V J 2032+4130}=(0.84 \pm 0.05) \times 10^{-12} \mathrm{~cm}^{-2} \mathrm{~s}^{-1}$. After processing the Cyg X-3 observation data, first by selection criteria associated with Cyg X-3 and then with $\mathrm{TeV}$ $\mathrm{J} 2032+4130$, we found that $8.1 \%$ of showers are common for both sources. Recognition of the source for each of the common showers is performed by analysing the angular distance of the arrival direction of these showers and the source coordinates. As a result, less than $4 \%$ of Cyg X-3 showers were recognized to be TeV J2032+4130 showers. This didn't change the average flux of Cyg X-3.

The differential $\gamma$-ray energy spectrum of $\mathrm{TeV}$ $\mathrm{J} 2032+4130$ in the energy range from 0.8 to $50 \mathrm{TeV}$ obtained by SHALON telescope is presented in Fig. 1. In the energy range $0.8-15 \mathrm{TeV}$ the differential spectrum of $\mathrm{TeV} \mathrm{J} 2032+4130$ is well described by the power law $F(E>0.8 \mathrm{TeV}) \propto E^{k}$, with $k=-1.73 \pm 0.10$ (see Fig. 1, top). Also, the source image in TeV-energies by SHALON are shown in Figure 1, bottom. Analysis of the $\gamma$-shower 

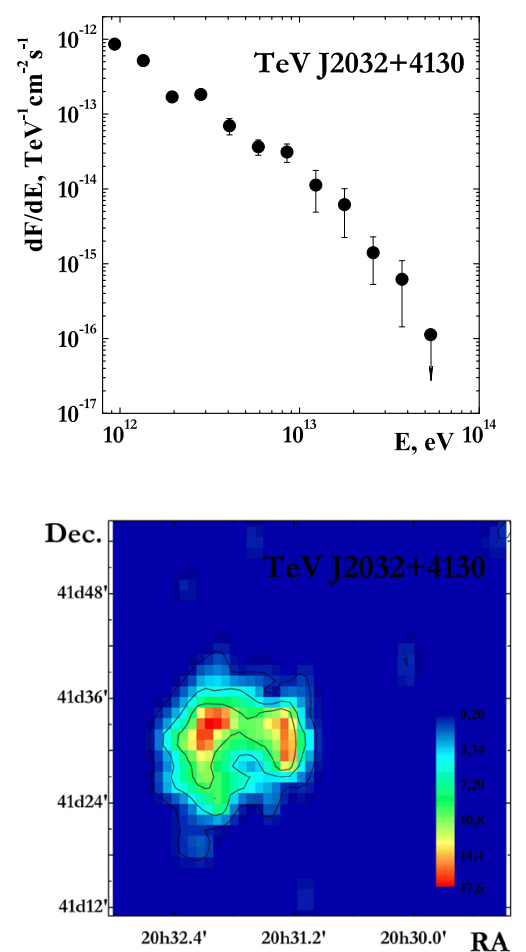

Figure 1. TeV J2032+4130 characteristics: top - differential gamma-ray spectrum in the energy range $1-50 \mathrm{TeV}$;

bottom - The image of TeV J2032+4130 at energies $>0.8 \mathrm{TeV}$ by SHALON

direction showed that the emission source is an extended area of $\sim 10^{\prime}$ size, coinciding with the experimental data of HEGRA [8], VERITAS [12] and MAGIC [13] (see the references, available from the references).

Very high energy data on the TeV J2032+4130 energy spectrum were obtained in different years with the HEGRA [8], VERITAS [12] and MAGIC [13] groundbased telescopes and with the MILAGRO [6] detector. Figure 2 shows the comparison of differential energy spectra of TeV J2032+4130 by SHALON (1995-2016), HEGRA(1999 - 2002), MAGIC (2005 - 2007), VERITAS (2009 - 2012) and data of MILAGRO (2011).

The differential spectrum was obtained with the VERITAS experiment in the energy region $700 \mathrm{GeV}-16 \mathrm{TeV}$. It is well fitted by a power law $d N / d E=C \times(E / 1 T e V)^{-\Gamma}$ with a spectral index of $\Gamma=2.1 \pm 0.14_{\text {stat }} \pm 0.21_{\text {sys }}$ and normalization at $1 \mathrm{TeV}$ of $C=\left(9.5 \pm 1.6_{\text {stat }} \pm 2.2_{\text {sys }}\right) \times 10^{-13}$ $\mathrm{TeV}^{-1} \mathrm{~cm}^{-2} \mathrm{sec}^{-1}$. with the $\chi^{2}=0.9$ per degree of freedom is $4.5 / 5=0.9$.

The differential spectrum of $\mathrm{TeV} \mathrm{J} 2032+4130$ obtained with MAGIC can be fitted by a power law function with the $\chi^{2}=0.9$ in the form of $d N / d E=(4.5 \pm 0.3) \times$ $10^{-13} \times(E / 1 T e V)^{-2.0 \pm 0.3}$.

The spectrum of TeV J2032+4130 in the energy range $1-10 \mathrm{TeV}$ was measured in HEGRA observations in the period 1999 - 2000. The shape is consistent with a power law $d N / d E=C \times(E / 1 T e V)^{-\Gamma}$ with the following parameters $C=\left(6.2 \pm 1.5_{\text {stat }} \pm 1.3_{\text {sys }}\right) \times 10^{-13} \mathrm{~cm}^{-2} \mathrm{sec}^{-1}$ and $\Gamma=1.9 \pm 0.1_{\text {stat }} \pm 0.3_{\text {sys }}$.

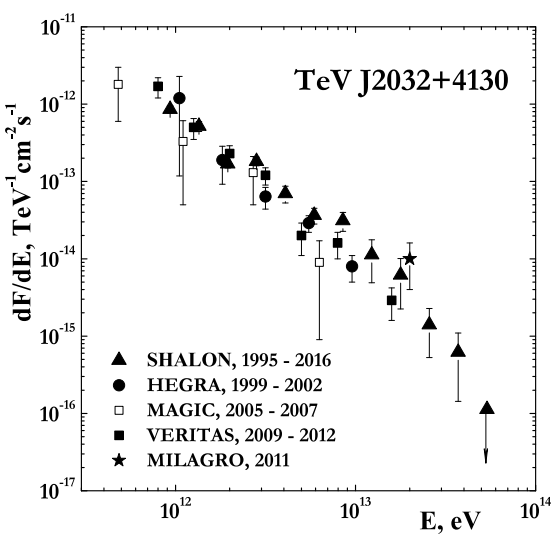

Figure 2. Differential spectrum of $\mathrm{TeV} J 2032+4130$ by SHALON ( $\mathbf{\Lambda}$ ) compared with HEGRA, VERITAS, MAGIC and MILAGRO experiments (see text).

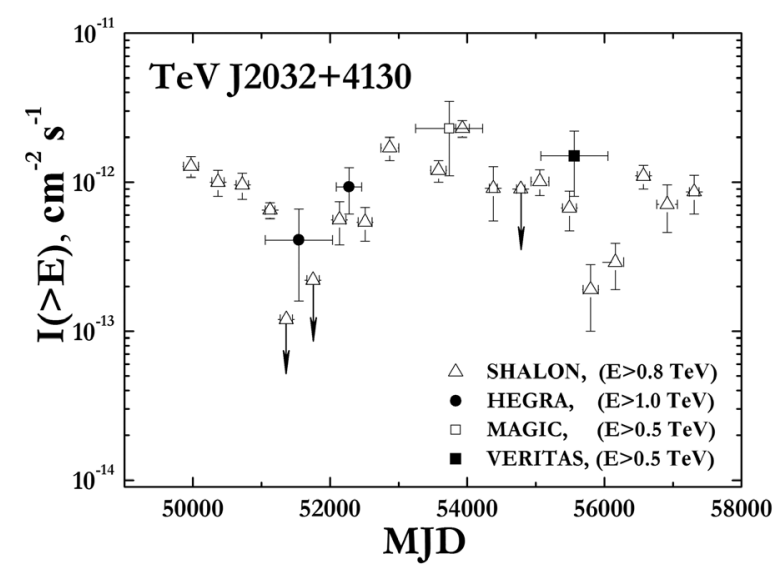

Figure 3. The light curve of TeV J2032+4130 at very high energies obtained in the long-term SHALON observations compared with HEGRA, MAGIC, and VERITAS experimental data.

The shape of the SHALON differential spectrum of gamma rays from $\mathrm{TeV} \mathrm{J} 2032+4130$ in the energy range from 0.8 to $35 \mathrm{TeV}$ fits well to a hard power law with an exponential cutoff (see. Fig. $2, \mathbf{\Delta}): d N / d E=(7.1 \pm 1.1) \times$ $10^{-13} \times\left(E_{\gamma} / 1 \mathrm{TeV}\right)^{-1.50 \pm 0.11} \times \exp \left(-E_{\gamma} /(16 \pm 3) \mathrm{TeV}\right)$ with the $\chi^{2} /$ Dof $=1.31$ where degree of freedom Dof $=8$.

The twenty-year-long SHALON observations of $\mathrm{TeV}$ $\mathrm{J} 2032+4130$ revealed flux variations at energies $>0.8$ TeV. Figure 3 shows the light curve of TeV J2032+4130 obtained in the SHALON experiment over the entire period of observations from 1995 to 2015 in comparison with the HEGRA, MAGIC, and VERITAS data. The white triangles indicate the integral fluxes from the SHALON data averaged over each year of observation. The integral fluxes for $\mathrm{TeV} \mathrm{J} 2032+4130$ from the data of other telescopes shown in Fig. 3 were determined in different time intervals: HEGRA (1999 - 2001, 2002), MAGIC (2005 2007) and VERITAS (2009 - 2012). The mean integral flux for TeV J2032+4130 at energies $>0.8 \mathrm{TeV}$ from the SHALON data was $(1.01 \pm 0.15) \times 10^{-12} \mathrm{~cm}^{-2} \mathrm{~s}^{-1}$ in the observation periods 1995 - 1997, 2003 - 2006, and 2013 - 2015; $(0.58 \pm 0.12) \times 10^{-12} \mathrm{~cm}^{-2} \mathrm{~s}^{-1}$ in 1998,2001 , 2002, 2010, and 2016; and a low flux, $(0.12 \pm 0.09) \times$ 

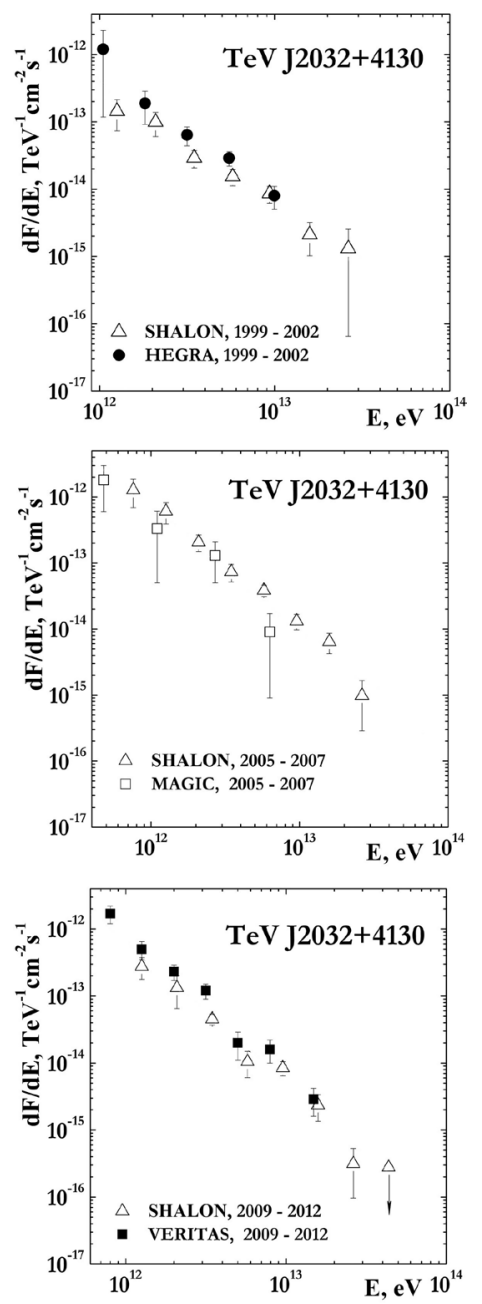

Figure 4. Differential spectra of $\mathrm{TeV} \mathrm{J} 2032+4130$ averaged over different periods of its observations from the SHALON data compared with the HEGRA (1999 - 2002), MAGIC (2005 2007), and VERITAS (2009 - 2012) experimental data.

$10^{-12} \mathrm{~cm}^{-2} \mathrm{~s}^{-1}$, was recorded in 1999, 2000, 2011, and 2012.

The differential spectra of TeV J2032+4130 from the SHALON data averaged over the corresponding periods of its observations in the HEGRA (1999 - 2002), MAGIC (2005 - 2007) and VERITAS (2009 - 2012) experiments are compared in Fig. 4.

\section{TeV J2032+413 nature}

The nature of the extended object $\mathrm{TeV}$ J2032+413 still remains unresolved. Since the discovery of TeV J2032+4130 several observations of the region have been made by X-ray telescopes Chandra, XMM-Newton which operate in the energy range of $0.1-10 \mathrm{keV}$ and 0.2 - $12 \mathrm{keV}$ respectively. Multiple point sources were detected in the region of TeV J2032+413 by Chandra, XMMNewton telescopes and then after the known point sources were subtracted XMM-Newton detected an extended Xray emission region (see $[12,18]$ ). Radio observations of the region have been made using the GMRT, yielding at least three sources of non-thermal emission along with an extended non-thermal diffuse emission. Moreover, VLA observations revealed shell-like structure of weak radio emission [18] consistent with the dimensions of the TeV source reported by HEGRA, VERITAS, MAGIC and SHALON.

\section{Conclusion}

The results of long-term observations of the $\mathrm{TeV}$ $\mathrm{J} 2032+4130$ object by the imaging atmospheric Cherenkov telescope SHALON are presented. The collected experimental data and refining of fluxes, spectrum shape and morphology of TeV J2032+413 can help in the future to determine an object type and to shed light on the source nature.

\section{References}

[1] A.A. Abdo, B. Allen, D. Berley et al., Astrophys. J. 658, L33 (2007)

[2] A.A. Abdo, M. Ackermann, M. Ajello et al., Science. 326, 1512 (2009)

[3] A.A. Abdo, M. Ackermann, M. Ajello et al., Astrophys. J. 187, 460 (2010)

[4] D.J. Thompson, D.L. Bertsch, B.L. Dingus et al., Astrophys. J. Suppl. Ser. 101, 259 (1995)

[5] J.A. Esposito, S.D. Hunter, G. Kanbach and P. Sreekumar, Astrophys. J. 461, 820 (1996)

[6] A.A. Abdo, B.T. Allen, T. Aune et al., Astrophys. J. 734, 28 (2011)

[7] J.H. Buckley, C.W. Akerlof, D.A. Carter-Lewis et al., Astron. Astrophys. 329, 639 (1998)

[8] F. Aharonian, A. Akhperjanian, M. Beilicke et al., Astron. Astrophys. 431, 197 (2005)

[9] V.G. Sinitsyna, in Proc. 16th European Cosmic Ray Symposium, ed. J. Medina, (Alcala de Henares: Universidad de Alcala, Spain), 383 (1998).

[10] V.G. Sinitsyna, V.Y. Sinitsyna, , Bulletin of the Lebedev Physics Institute, (New York: Allerton Press, Inc.), 40(5), 113 (2013).

[11] V.G. Sinitsyna and V.Y. Sinitsyna, EPJ Web of Conferences 145, 04002 (2017)

[12] E. Aliu, T. Aune, B. Behera et al., Astrophys. J. 783, 16 (2014)

[13] J. Albert, E. Aliu, H. Anderhub et al., Astrophys. J. 675, L25 (2008)

[14] V.G. Sinitsyna Nuovo Cimento 19C(6), 965 (1996)

[15] V.G. Sinitsyna, V. Yu.. Sinitsyna, Astr. Lett. 40(2-3), 75 (2014)

[16] V.G. Sinitsyna et al., Adv. Space Res. doi:10.1016/j.asr.2017.04.007 (2017)

[17] T.-P. Li and Y.-Q. Ma, Astrophys. J. 272, 317 (1983)

[18] Y.M. Butt, J.A. Combi, J. Drake et al., Mon. Not. Roy. Astron. Soc. 385, 1764 (2008) 